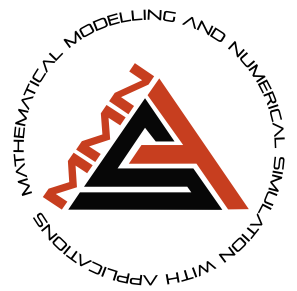

\title{
Three-dimensional fractional system with the stability condition and chaos control
}

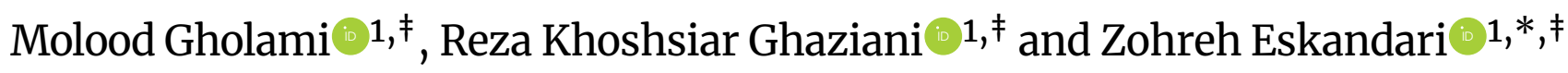 \\ ${ }^{1}$ Department of Mathematical Sciences, Shahrekord University, Shahrekord, Iran \\ *Corresponding Author \\ ${ }^{\ddagger}$ moloodgholami1370@gmail.com (Molood Gholami); khoshsiar@sci.sku.ac.ir (Reza Khoshsiar Ghaziani); z.eskandari@sku.ac.ir \\ (Zohreh Eskandari)
}

\begin{abstract}
A three-dimensional system is introduced in this paper and its local stability is analyzed. Our study establishes the validity and uniqueness of the linear feedback control for the proposed system and proves its existence and uniqueness. The numerical simulation algorithm described by Atanackovic and Stankovic is finally applied. The analytical results are analyzed and the dynamics of the system are explored in more detail.
\end{abstract}

Key words: Fractional-order system; stability; control; chaotic system

AMS 2020 Classification: 34A08; 34C23;34H10

\section{Introduction}

Modeling and investigating actual phenomena can be accomplished with fractional dynamical systems. A dynamical system may exhibit chaos as one of its important dynamics. Dynamical chaos disappears when the fractional order falls below a threshold in a fractional-order chaotic system. There have been several articles discussing the minimum effective dimension below which the system remains chaotic, $[1,2,3,4,5,6,7]$.

Chaos theory is a field of mathematics that has already attracted the attention of many researchers from different fields of science, engineering and medicine. Chaos theory describes the behavior of certain dynamical systems whose state evolves with time and are highly sensitive to initial conditions. Because of the complexity of chaotic behavior in dynamical systems, it finds applications in a variety of fields, such as science, technology and medicine $[8,9,10,11,12,13,14]$. Studying chaotic systems can be a very valuable endeavor. Sene [15] in his paper studied the applications of the fractional-order chaotic system in the sense of Caputo fractional derivative. The presence or absence of chaotic behaviors of their model was presented in terms of the Lyapunov exponents. For the model description, the circuit schematic was drawn and simulated. Naik et al. [16] in their paper studied the chaotic dynamics of a fractional-order cancer model. A detailed analysis of the equilibrium points was also considered. They also calculated the Lyapunov exponents that give the existence of chaotic behavior of the model.

Leibniz in 1695 was the first to introduce the fractional calculus followed by Liouville in 1834, Riemann in 1892 and others [17]. Fractional calculus represents the generalization of integrals and derivatives to non-integer order. After Leibniz fractional calculus has gained increasing popularity and finds applications in various fields of science, technology and medicine [18, 19, 20, 21, 22, 23]. Recently, Ozkose and Yavuz [24] in their paper studied in fractional-order case the relations between COVID-19 and diabetes diseases under the hereditary traits then validated their model by the real data from Turkey. The Adams-Bashforth-Moulton predictor-corrector method was employed for the numerical solution of their model. For the advantages of the fractional-order derivative, they considered the memory trace and hereditary traits in the model. Fractional model, similar electrode-electrolyte, electromagnetic, and wave models have been found to

> Received: 04.11.2021 > Revised: 05.02.2022 > Accepted: 08.02.2022 > Published: 16.02.2022 
explain many systems in the physical, chemical, and biological processes.

The rest of the paper is decorated as: after the introduction in Section 1, Section 2 gives some preliminaries and discusses a chaotic three-dimensional system. Section 3 examines whether or not a proposed system solution exists and is unique. In Section 4, we introduced the stability conditions of the equilibrium points of the system. The linear feedback control system studied in Section 5 is based on the Routh-Hurwitz method. In Section 6, we present numerical simulations based on algorithmic methods and discuss the obtained results. Finally, in Section 7, we conclude the study.

\section{Preliminaries}

Many well known fractional derivatives including Riemann-Liouville, Grunwald-Letnikov as well as Caputo exist in the literature and are all common fractional derivative definitions. As a result, we investigate the fractional derivative of Caputo, as defined in [25]:

$$
\begin{aligned}
D^{\eta} g(\tau) & =\frac{1}{\Gamma(m-\eta)} \int_{0}^{t}(\tau-\sigma)^{m-\eta-1} g^{(m)}(\sigma) d \sigma \\
& =j^{m-\eta}\left(\frac{d^{m}}{d \tau^{m}} g(\tau)\right) .
\end{aligned}
$$

It is defined as follows: $m$ is integer, $m-1<\eta<m$ and $\Gamma$ is the Gamma function, and $j^{\theta}$ is Riemann-Liouville integral operator.

$$
j^{\theta} g(\tau)=\frac{1}{\Gamma(\theta)} \int_{0}^{t}(\tau-\sigma)^{\theta-1} g^{(m)}(\sigma) d \sigma, \quad \tau>0 .
$$

Theorem 1 [26] An autonomous linear system

$$
D^{\eta} x=L x, \quad x(0)=x_{0}
$$

where $L$ is a $m \times m$ matrix and $0<\eta<1$ is asymptotically stable if and only if $|\arg (\mu)|>\frac{\eta \pi}{2}$ for all eigenvalues $\mu$ of $L$. The components of the solution $x(\tau)$ decay to zero in this case, each component of solution $x(\tau)$ decays toward or like $\tau^{-\eta}$. Also, this linear system is stable if and only if $|\arg (\mu)| \geq \frac{\eta \pi}{2}$ and those critical eigenvalues that satisfy $\operatorname{larg}(\mu) \mid=\frac{\eta \eta \pi}{2}$ that geometric multiplication is one. In Ref. [27], a chaotic system in three dimensions is described by:

$$
\left\{\begin{array}{l}
x_{1}^{\prime}(\tau)=a x_{1}-x_{2} x_{3}, \\
x_{2}^{\prime}(\tau)=-b x_{2}+x_{1} x_{3}, \\
x_{3}^{\prime}(\tau)=-c x_{3}+x_{1}^{2},
\end{array}\right.
$$

where $x_{1}, x_{2}, x_{3}$ are state variables, $a, b, c \in \mathbb{R}^{+}$are constant parameters. For $a=6, b=12, c=14$, the chaotic attractors for system (4) are displayed in Fig. 1.

Three equilibrium points exist in the system

$$
O=(0,0,0), \quad Q_{1}=\left(\sqrt[4]{a b c^{2}}, \sqrt[4]{a^{3} c^{2} / b}, \sqrt{a b}\right), \quad Q_{2}=\left(-\sqrt[4]{a b c^{2}},-\sqrt[4]{a^{3} c^{2} / b},-\sqrt{a b}\right) .
$$

\section{Solution's existence and uniqueness}

Taking into account the initial value problem:

$$
D^{\eta} W(\tau)=g(\tau, W(\tau)), \quad 0<\tau<\Omega, \quad W^{(k)}(0)=W_{0}^{(k)}, \quad k=0,1, \ldots, m-1
$$

Theorem 2 (Existence [28]) Let us consider

$$
E:=\left[0, W^{*}\right] \times\left[W_{0}^{(0)}-\varepsilon, W_{0}^{(0)}+\varepsilon\right]
$$

with some $W^{*}>0$ and some $\varepsilon>0$, and the function $g: E \rightarrow \mathbb{R} \chi:=\min \left\{\chi^{*},\left(\varepsilon \Gamma(\eta+1) /\|g\|_{\infty}^{\frac{1}{\eta}}\right\}\right.$. Then, there exists a function $W:[0, \chi] \rightarrow \mathbb{R}$ which solves the initial value problem (5).

Theorem 3 (Uniqueness [28]) Let us consider

$$
E:=\left[0, \chi^{*}\right] \times\left[W_{0}^{(0)}-\varepsilon, W_{0}^{(0)}+\varepsilon\right]
$$

with some $\chi^{*}>0$ and some $\varepsilon>0$. Therefore the function $f: E \rightarrow \mathbb{R}$ is surrounded on $E$ and regard the second variable, meet the Lipschitz condition, i.e.,

$$
|g(\tau, W)-g(\tau, V)| \leq J|W-V|
$$



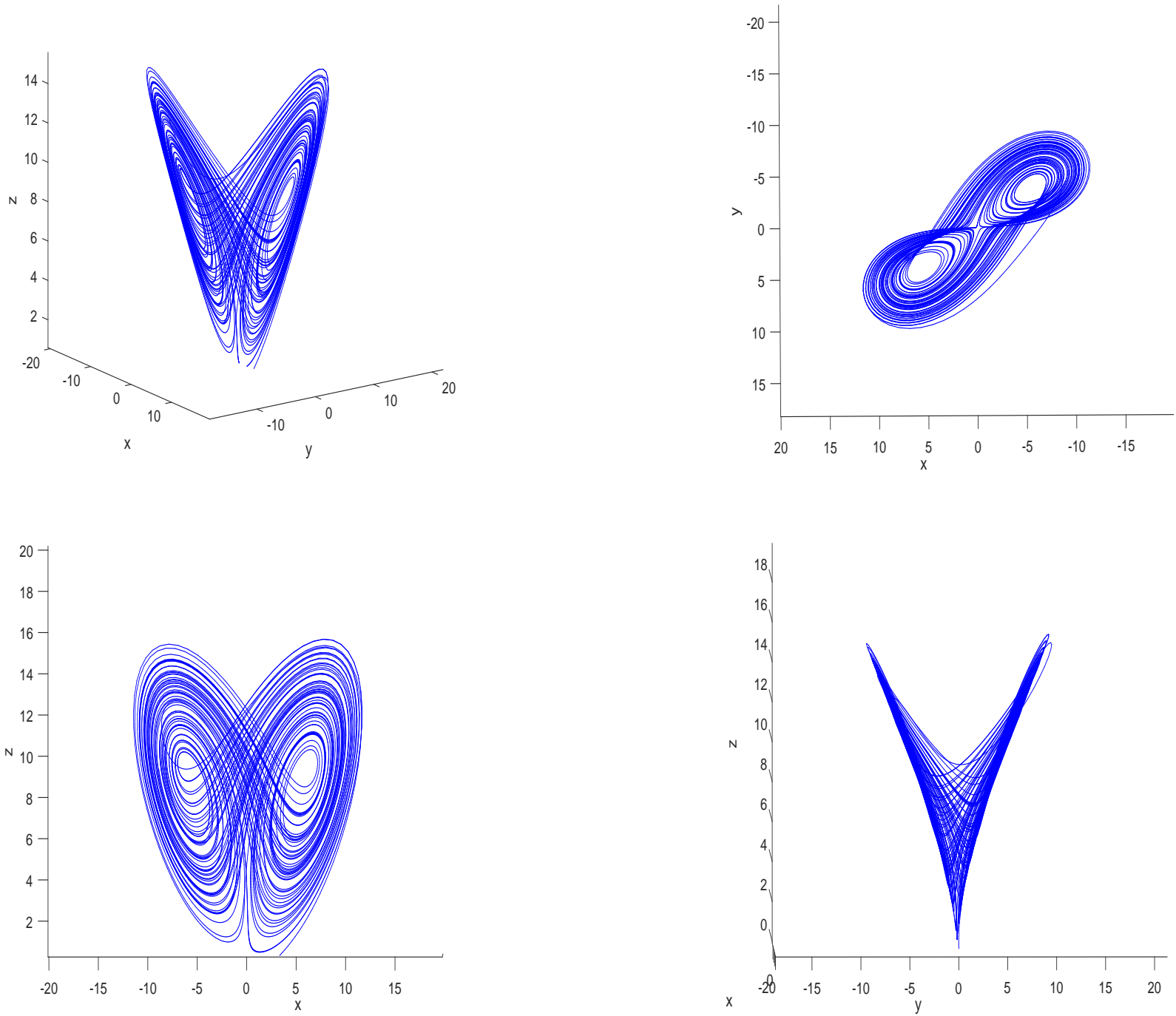

Figure 1. The chaotic attractors for system (4)

with constant $J>0$ independent $\tau, W$ and $V$. Then, describe $\chi$ as Theorem (2), there exists one function $W:[0, \chi] \rightarrow \mathbb{R}$ solving the initial value problem (5).

Theorem 4 In the fractional-order three-dimensional system (4), the initial value problem can be expressed as follows:

$$
D^{\eta} x(\tau)=A x(\tau)+x_{1}(\tau) B x(\tau)+x_{2}(\tau) C x(\tau), \quad x(0)=x_{0},
$$

where $0<\tau<\Omega, x(\tau)=\left(x_{1}(\tau), x_{2}(\tau), x_{3}(\tau)\right)^{\Omega} \in \mathbb{R}^{3}, x(0)=\left(x_{10}, x_{20}, x_{30}\right)$

$$
A=\left(\begin{array}{ccc}
a & 0 & 0 \\
0 & -b & 0 \\
0 & 0 & -c
\end{array}\right), \quad B=\left(\begin{array}{ccc}
0 & 0 & 0 \\
0 & 0 & 1 \\
1 & 0 & 0
\end{array}\right), \quad C=\left(\begin{array}{ccc}
0 & 0 & -1 \\
0 & 0 & 0 \\
0 & 0 & 0
\end{array}\right)
$$

If

$$
\Omega:=\min \left\{\Omega^{*},\left(\varepsilon \Gamma(\eta+1) /\|g\|_{\infty}\right)^{\frac{1}{\eta}}\right\}, \quad \Omega^{*}>0,
$$

there exists a unique solution for (6).

Proof Consider

$$
g(x(\tau))=A x(\tau)+x_{1}(\tau) B x(\tau)+x_{2}(\tau) C x(\tau), \quad x(\tau) \in\left[0, \Omega^{*}\right] \times\left[x_{0}-\varepsilon, x_{0}+\varepsilon\right]
$$


for any $\Omega^{*}, \varepsilon>0$. Moreover, one has

$$
\begin{aligned}
|g(x)-g(y)| & =\left|A(x-y)+x_{1} B x-y_{1} B y+x_{2} C x-y_{2} C y\right| \\
& \leq|| A|||(x-y)|+\left|x_{1} B x-y_{1} B y\right|+\left|x_{2} C x-y_{2} C y\right|
\end{aligned}
$$

where $y(\tau) \in\left[0, \Omega^{*}\right] \times\left[x_{0}-\varepsilon, x_{0}+\varepsilon\right]$, and | | | represents the matrix vector norm. Clearly

$$
\begin{aligned}
\left|x_{1} B x-y_{1} B y\right| & =\left|x_{1} B x-y_{1} B x+y_{1} B x-y_{1} B y\right| \\
& =\mid(x-x)-) B x+y_{1} B(x-y) \mid \\
& \leq|| B||\left(|x|+\left|y_{1}\right|\right)(|x-y|) .
\end{aligned}
$$

Likewise, one has the following result:

$$
\left|x_{2} C x-y_{2} C y\right| \leq|| C||\left(|x|+\left|y_{2}\right|\right)(|x-y|)
$$

Eq. (7) gives the result that

$$
\begin{aligned}
|g(x)-g(y)| & \leq\left[|| A||+|| B||\left(|x|+\left|y_{1}\right|\right)+|| C||\left(|x|+\left|y_{2}\right|\right)\right](|x-y|) \\
& \leq\left[|| A||+(|| B||+|| C||)\left(2\left|x_{0}\right|+2 \epsilon\right)\right](|x-y|) \\
& \leq L(|| x-y||)
\end{aligned}
$$

where $J=|| A||+(|| B||+|| C||)\left(2\left|x_{0}\right|+2 \varepsilon\right)>0$. Hence, the fractional-order dimensional system is Lipschitz-satisfying. Then, compatible to the existence and uniqueness theorem of the fractional-order dimensional system the initial value problem of the commensurate order system (6) has a unique solution in the interval

$$
\left.\Omega:=\min \left\{\Omega^{*},\left(\varepsilon \Gamma(\eta+1) /\|g\|_{\infty}\right)\right)^{\frac{1}{\eta}}\right\}
$$

\section{Conditions for the stability of equilibrium points}

The characteristic equation of system (4) is determined by

$$
p(\mu)=\mu^{3}+r_{1} \mu^{2}+r_{2} \mu+r_{3}=0,
$$

whose discriminant $D(p)$ is defined by

$$
D(p)=R\left(p, p^{\prime}\right),
$$

and

$$
D(p)=18 r_{1} r_{2} r_{3}+r_{1}^{2} r_{2}^{2}-4 r_{3} r_{1}^{3}-4 r_{2}^{3}-27 r_{3}^{3}
$$

If $\Delta_{1}, \Delta_{2}$ and $\Delta_{3}$ are Routh-Hurwitz determinants $\Delta_{1}=r_{1}, \Delta_{2}=\left|\begin{array}{cc}r_{1} & 1 \\ r_{3} & r_{2}\end{array}\right|$ and $\Delta_{3}=r_{3}$. Thus we have the following stability conditions [28].

(I) If $D(p)<0, a_{1}>0, a_{2}>0, a_{1} a_{2}=a_{3}$, then the equilibrium point is locally asymptotically stable for all $\eta \in(0,1)$.

(II) The condition $r_{3}>0$, is the necessary condition for the equilibrium point to be locally asymptotically stable.

Some stability conditions for the equilibrium points $Q_{0}, Q_{1}$ and $Q_{2}$

The characteristic polynomial of equilibrium point $Q_{0}$ is given by:

$$
p(\mu)=\mu^{3}+\mu^{2}(c+b-a)+\mu(b c-a c)-a b c .
$$

It is clear that $r_{3}=-a b c<0$, thus applying the stability condition (II) to characteristic equation (11) implies that $E_{0}$ in unstable. Similary, the equilibrium point $Q_{1}$ and $Q_{2}$ have the same characteristic polynomial, which given as:

$$
p(\mu)=\mu^{3}+(c+b-a) \mu^{2}+(a c+b c) \mu+4 a b c=0 .
$$

Thus, applying the Routh-Hurwitz conditions and the necessary stability condition part (I), imply that equilibrium points $Q_{1}, Q_{2}$ are unstable. 


\section{Linear feedback control of the chaotic system}

Here, the control of fractional three-dimensional chaotic system (4) is discussed by using the linear feedback control.

The controlled fractional order chaotic system (4) is given by:

$$
\left\{\begin{array}{l}
D^{\eta} x_{1}(t)=a x_{1}-x_{2} x_{3}-k_{1}\left(x_{1}-\bar{x}_{1}\right) \\
D^{\eta} x_{2}(t)=-b x_{2}+x_{1} x_{3}-k_{2}\left(x_{2}-\bar{x}_{2}\right) \\
D^{\eta} x_{3}(t)=-c x_{3}+x_{1}^{2}-k_{3}\left(x_{3}-\bar{x}_{3}\right),
\end{array}\right.
$$

where $\left(k_{1}, k_{2}, k_{3}\right)$ are feedback control and $k_{1}, k_{2}, k_{3}>0$ and by suitable choice of feedback control according to stability conditions (I, II), we can drive the system (13) trajectories to unstable equilibrium point $Q_{1}$.

\section{Controlling chaos for the equilibrium point $Q_{1}$}

In this section, we apply stability condition of chaotic system to study chaos control. For this, we obtain the characteristic equation of the controlled system (13) evaluated at the equilibrium point by:

$$
\mu^{3}+\mu^{2}\left(s_{1}+s_{2}+k_{2}+b\right)+\mu\left(s_{1} s_{2}+2 b s_{2}+k_{2} s_{1}+k_{2} s_{2}\right)+\left(s_{1} s_{2}\right) k_{2}+b s_{1}\left(c+k_{2}\right),
$$

where $s_{1}=\left(k_{1}-a\right)$ and $s_{2}=\left(k_{3}+c\right)$. By applying the Routh-Hurwitz conditions (I, II) to equilibrium point (13) we find that: $k_{1}, k_{2}, k_{3}$, are all positive and defined by equation (13).

Furthermore, the inequality is enough conditions for stabilizing the controlled system (13) to the equilibrium point $Q_{1}$ and $Q_{2}$ : In the system (13) we consider the fixed parameters $a=6, b=12, c=4$ and using the feedback control gains $\left(k_{1}, k_{2}, k_{3}\right)=(2.88,1.33,0.52)$.

For the above mentioned value parameters and feedback control gains, it becomes clear that the trajectories of controlled system (13) with fractional order $\eta$ converge to the equilibrium point $Q_{1}$.

Fig. 2 shows the trajectories of controlled fractional system for $\eta=0.98$, which converges to the equilibrium point $Q_{1}$. However, when $\eta=1$ the controlled system (13) is not stable near equilibrium point $Q_{1}$ (see Fig. 3).

\section{Numerical simulations and discussion}

For solving system (4), we employ a numerical technique developed by Atanackovic and Stankovic [29] to solve the fractional differential equation, and we depict trajectories of system (4) using the well known Runge-Kutta method of order fourth for parameters $a=6, b=12$, $c=4$. We have the equilibrium point $Q_{1}=(135.764,67.882,8.485)$ and $Q_{2}=(-135.764,-67.882,-8.485)$.

Fig. 1 presents numerical values for system (4) for different values. An analysis of the local stability of a three-dimensional system is presented in this paper. This study aims to verify the validity and uniqueness of linear feedback control for the proposed system and prove its existence and uniqueness. An algorithm is finally applied to the numerical simulation.

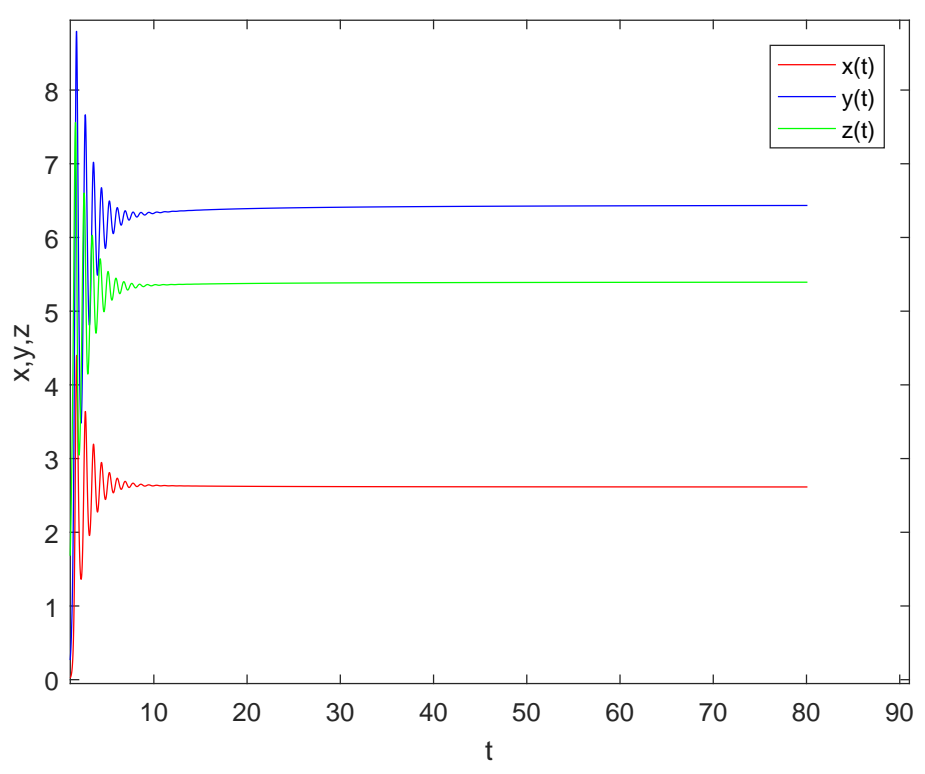

Figure 2. The trajectories of the controlled system (13) by $\eta=0.98$ 


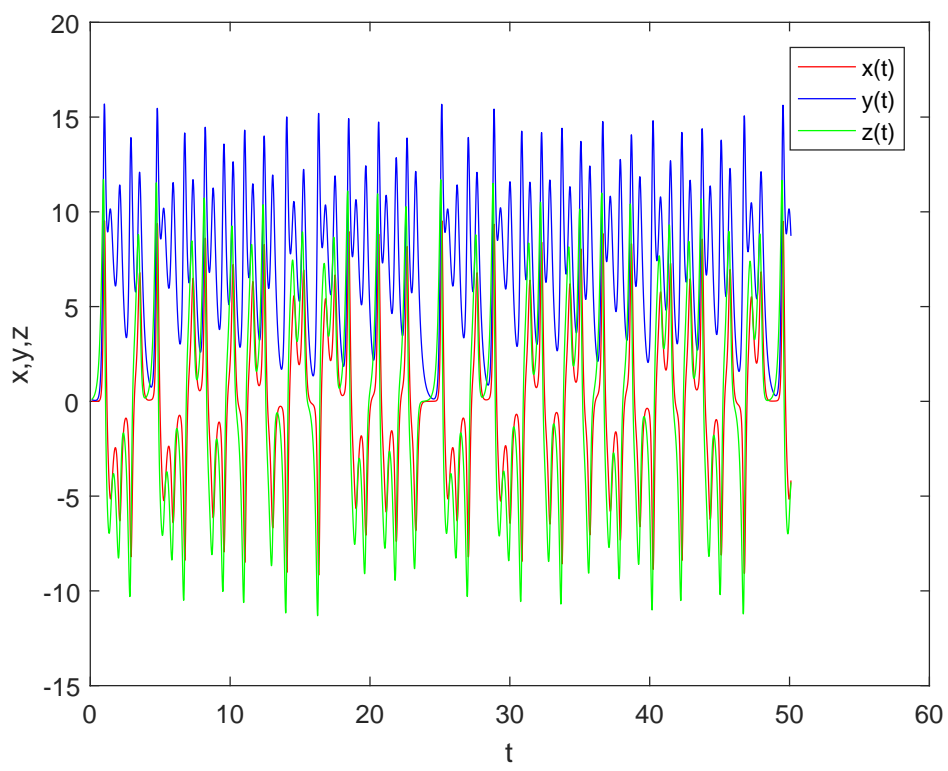

Figure 3. The trajectories of the controlled system (13) by $\eta=1$

\section{Conclusions}

In the present paper, we examined a three-dimensional fractional order chaotic system. The conditions that ensure the existence and uniqueness of its solution were identified. We employed Routh-Hurwitz method to determine the stability conditions. We also employed the feedback control of the chaotic system with fractional order. Through the numerical simulations, the performance and authenticity of the proposed method were presented. The trajectories of the model (4) through the well known Runge-Kutta method of order fourth were depicted. It is concluded from the obtained results that the fractional power of the derivative has a significant effect on the dynamic process. Also, it is observed that the smaller fractional power of the derivative is the chaotic behavior of the system.

\section{Declarations}

\section{Consent for publication}

Not applicable.

\section{Conflicts of interest}

The authors declare that they have no conflict of interests.

\section{Funding}

Not applicable.

\section{Author's contributions}

M.G.: Conceptualization, Methodology, Software, Writing-Original draft. R.K.G.: Conceptualization, Methodology, Supervision, Investigation. Z.E.: Software, Methodology, Data Curation. All authors discussed the results and contributed to the final manuscript.

\section{Acknowledgements}

Not applicable.

\section{References}

[1] Allegretti, S., Bulai, I.M., Marino, R., Menandro, M.A., \& Parisi, K. Vaccination effect conjoint to fraction of avoided contacts for a Sars-Cov-2 mathematical model. Mathematical Modelling and Numerical Simulation with Applications, 1(2), 56-66, (2021). [CrossRef]

[2] Özköse, F., Şenel, M.T., \& Habbireeh, R. Fractional-order mathematical modelling of cancer cells-cancer stem cells-immune system interaction with chemotherapy. Mathematical Modelling and Numerical Simulation with Applications, 1(2), 67-83, (2021). [CrossRef]

[3] Ikram, R., Khan, A., Zahri, M., Saeed, A., Yavuz, M., \& Kumam, P. Extinction and stationary distribution of a stochastic COVID-19 epidemic model with time-delay. Computers in Biology and Medicine, 141, 105115, (2022). [CrossRef] 
[4] Özköse, F., Yılmaz, S., Yavuz, M., Öztürk, İ., Şenel, M.T., Bağcl, B.Ş., ... \& Önal, Ö. A Fractional Modeling of Tumor-Immune System Interaction Related to Lung Cancer with Real Data. The European Physical Journal Plus, 137(1), 1-28, (2022). [CrossRef]

[5] Oud, M.A.A., Ali, A., Alrabaiah, H., Ullah, S., Khan, M.A., \& Islam, S. A fractional order mathematical model for COVID-19 dynamics with quarantine, isolation, and environmental viral load. Advances in Difference Equations, 2021(1), 1-19, (2021). [CrossRef]

[6] Ali, A., Alshammari, F.S., Islam, S., Khan, M. A., \& Ullah, S. Modeling and analysis of the dynamics of novel coronavirus (COVID-19) with Caputo fractional derivative. Results in Physics, 20, 103669, (2021). [CrossRef]

[7] Ali, A., Islam, S., Khan, M.R., Rasheed, S., Allehiany, F.M., Baili, J., ... \& Ahmad, H. Dynamics of a fractional order Zika virus model with mutant. Alexandria Engineering Journal, 61(6), 4821-4836, (2021). [CrossRef]

[8] Hammouch, Z., Yavuz, M., \& Özdemir, N. Numerical solutions and synchronization of a variable-order fractional chaotic system. Mathematical Modelling and Numerical Simulation with Applications, 1(1), 11-23, (2021). [CrossRef]

[9] Daşbaşı, B. Stability analysis of an incommensurate fractional-order SIR model. Mathematical Modelling and Numerical Simulation with Applications, 1(1), 44-55, (2021). [CrossRef]

[10] Naik, P.A., Yavuz, M., Qureshi, S., Zu, J., \& Townley, S. Modeling and analysis of COVID-19 epidemics with treatment in fractional derivatives using real data from Pakistan. The European Physical Journal Plus, 135(10), 1-42, (2020). [CrossRef]

[11] Naik, P.A., Owolabi, K.M., Yavuz, M., \& Zu, J. Chaotic dynamics of a fractional order HIV-1 model involving AIDS-related cancer cells. Chaos, Solitons \& Fractals, 140, 110272, (2020). [CrossRef]

[12] Naik, P.A. Global dynamics of a fractional-order SIR epidemic model with memory. International Journal of Biomathematics, 13(08), 2050071, (2020). [CrossRef]

[13] Alidousti, J., \& Eskandari, Z. Dynamical behavior and Poincare section of fractional-order centrifugal governor system. Mathematics and Computers in Simulation, 182, 791-806, (2021). [CrossRef]

[14] Naik, P.A., Eskandari, Z., \& Shahraki, H.E. Flip and generalized flip bifurcations of a two-dimensional discrete-time chemical model. Mathematical Modelling and Numerical Simulation with Applications, 1(2), 95-101, (2021). [CrossRef]

[15] Sene, N. Study of a Fractional-Order Chaotic System Represented by the Caputo Operator. Complexity, 2021, Article ID 5534872, (2021). [CrossRef]

[16] Naik, P.A., Zu, J. \& Naik, M. Stability analysis of a fractional-order cancer model with chaotic dynamics. International Journal of Biomathematics, 14(6), 2150046, (2021). [CrossRef]

[17] Magin, R. Fractional calculus in bioengineering. Critical Reviews in Biomedical Engineering, 32(1), 1-104, (2004). [CrossRef]

[18] Naik, P.A., Yavuz, M. \& Zu, J. The role of prostitution on HIV transmission with memory: A modeling approach. Alexandria Engineering Journal, 59(4), 2513-2531, (2020). [CrossRef]

[19] Naik, P.A., Zu, J. \& Owolabi, K. Global dynamics of a fractional order model for the transmission of HIV epidemic with optimal control. Chaos Solitons \& Fractals, 138, 109826, (2020). [CrossRef]

[20] Naik, P.A., Owolabi, K.M, Zu, J. \& Naik, M. Modeling the transmission dynamics of COVID-19 pandemic in Caputo type fractional derivative. Journal of Multiscale Modelling, 12(3), 2150006-107, (2021). [CrossRef]

[21] Yavuz, M. \& Sene, N. Fundamental calculus of the fractional derivative defined with Rabotnov exponential kernel and application to nonlinear dispersive wave model. Journal of Ocean Engineering and Science, 6(2), 196-205, (2021). [CrossRef]

[22] Yavuz, M., Sulaiman, T.A., Usta, F. \& Bulut, H. Analysis and numerical computations of the fractional regularized long-wave equation with damping term. Mathematical Methods in the Applied Sciences, 44(9), 7538-7555, (2021). [CrossRef]

[23] Sene, N. Second-grade fluid with Newtonian heating under Caputo fractional derivative: analytical investigations via Laplace transforms. Mathematical Modelling and Numerical Simulation with Applications, 2(1), 13-25, (2022). [CrossRef]

[24] Ozkose, F. \& Yavuz, M. Investigation of interactions between COVID-19 and diabetes with hereditary traits using real data: A case study in Turkey. Computers in Biology and Medicine, 141, 105044, (2022). [CrossRef]

[25] Caputo, M. Linear models of dissipation whose Q is almost frequency independent-II. Geophysical Journal International, 13(5), 529-539, (1967). [CrossRef]

[26] Matignon, D. (1996, July). Stability results for fractional differential equations with applications to control processing. In Computational engineering in systems applications (Vol. 2, No. 1, pp. 963-968).

[27] Lai, Q., \& Wang, L. Chaos, bifurcation, coexisting attractors and circuit design of a three-dimensional continuous autonomous system. Optik, 127(13), 5400-5406, (2016). [CrossRef]

[28] Ahmed, E., El-Sayed, A.M.A., \& El-Saka, H.A. On some Routh-Hurwitz conditions for fractional order differential equations and their applications in Lorenz, Rössler, Chua and Chen systems. Physics Letters A, 358(1), 1-4, (2006). [CrossRef]

[29] Atanackovic, T.M., \& Stankovic, B. On a numerical scheme for solving differential equations of fractional order. Mechanics Research Communications, 35(7), 429-438, (2008). [CrossRef]

Mathematical Modelling and Numerical Simulation with Applications (MMNSA) (https://www.mmnsa.org)

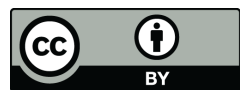

Copyright: ( 2022 by the authors. This work is licensed under a Creative Commons Attribution 4.0 (CC BY) International License. The authors retain ownership of the copyright for their article, but they allow anyone to download, reuse, reprint, modify, distribute, and/or copy articles in MMNSA, so long as the original authors and source are credited. To see the complete license contents, please visit (http://creativecommons.org/licenses/by/4.0/). 